\title{
Location Based Reminder System with Remote Request
}

\author{
Anisha Sinha \\ D.Y Patil Institute of Engg. \\ Ambi \\ Pune, Maharashtra, India
}

\author{
Aditi Joshi \\ D.Y Patil Institute of Engg. \\ Ambi \\ Pune, Maharashtra, India
}

\author{
Tanay Kale \\ D.Y Patil Institute of Engg. \\ Ambi \\ Pune, Maharashtra, India
}

\begin{abstract}
We all being human usually forget some things which we have to get from other. In such a case, this application will be useful to remember the things. Location-based alarm using GPS is an attempt to add an alarm facility for mobiles, based on the location of the device and to set a to-do list that is the task to be performed. The location-based alarm will give you an alert and a notification when you reach your desired destination. Moreover, we are up with a dynamic approach of remote handling for reminder system. We have put forth an idea where in user can connect and handle each other's reminder system. Hence when you forget of setting reminder itself, your connected friend may set it for you.
\end{abstract}

Keywords: GPS (Global Positioning System), LBS (Location Based Services), Location Based Reminder System

\section{INTRODUCTION}

Technology and its future developments are basically the approaches to finding ways to make life easier. A good product is judged by its simplicity, practicality, and functionality. Mobile information and communication technology determine society and behaviors' since it represents an element of individual quality and a way of communicating and doing business. The potential of hardware is such that it's difficult to make a proper use of it. By adapting to the users' requirements through improvement in the software side, hardware's capacity can be justified for a bit. The mobile applications focus on the technology, hence they are developed for certain purpose or for special technologies. The main aim of location-based services is to notify the customer of particular things based on their current location. We as human beings have a tendency to often forget things hence some of the important work to be done remains incomplete. We have location-based services at our disposal to overcome this shortcoming. However, some of the current location based services fall short on some part as they do not efficiently use the information; services are provided to the customer irrespective of their intention and current location. Thus, to overcome such situation we have proposed a location-based reminder system. This project is similar to an alarm, but the main and the most important difference is that it is not just a time alarm or task reminder but a location prompt. It comes with a provision of setting an alarm for a particular plan. The location-based alarm is a GPS based alarm, If a reminder is set for a particular locale, it will prompt with notification once it detects you are within the user-defined range from the destination. The users' current location will be saved in terms of longitudes and latitudes. The reminder will work when the user gets closer to the location. This locationbased alarm is useful for the traveling salespersons and passengers traveling in trains/buses. It is not an easy task to remember of all task and optimal routs for a traveling salesperson. So by using this application, he can set an alarm to the places, where he needs to go. It works wonders as you will be given a reminder to do/buy things at the exact location rather than keep the reminder on all day. Here also a new additional functionality is provided; setting of the radius. If the user chooses $500 \mathrm{~m}$ as the radius, then the alarm will go off when the user is in the proximity of the set location but $500 \mathrm{~m}$ meters away from it.

\section{ANDROID SYSTEM: OVERVIEW}

Android is an operating system basically for smart phones based on a modified version of Linux. Android OS is a stack of software components having five main sections and four main layers:

\subsection{Linux Kernel}

Linux Kernel is at the bottom of the layers with approximately 115 patches providing a level of abstraction between the device hardware and containing all the essential hardware drivers like camera, keypad, display etc. The kernel handles networking and a vast array of device drivers.

\subsection{Libraries}

Libraries including open-source Web browser engine WebKit and several android libraries : android.app, android.content, android.database, android.opengl, android.os, android.text, android.view, android.widget and android.webkit.

\subsection{Android Run-time}

It provides a key component called (DVM) Dalvik Virtual Machine which is a kind of (JVM) Java Virtual Machine specially designed and optimized for Android.

\subsection{Application Framework}

It provides many higher-level services to applications including: Activity Manager controlling all aspects of lifecycle and activity stack; Content Providers allowing publishing and sharing of data; Resource Manager providing an access to non-code embedded resources; Notifications Manager displaying alerts and notifications; 
View System which is an extensible set of views to create application UI.

\subsection{Application}

All the Android applications are present and installed on this layer only.

\section{IMPLEMENTATION}

This application includes five modules:

\subsection{Geo-Fencing Model}

In the Geo-fencing model the GPS system is used for getting the current location and also the location for which the alarm is to be set. Google Server is a web mapping service application and technology provided by Google, that powers many map-based services, including the Google Maps website and maps embedded on third-party websites via the Google Maps API. There is a provision of street maps along with route planner for various traveling modes (walking, twowheeler, four-wheeler, public transportation) including a locator for urban businesses in numerous countries around the world. Google Maps for Mobile introduced a Java application called Google Maps for Mobile, intended to run on any Android-based phone or mobile device. Many of the web-based site's features are provided in the application. The software looks up the location of the cell site using a database of known wireless networks and site.

\subsection{Set Reminder for Location and Task}

The user location is taken as input as soon as the location alert setting system is desired to be activated.. This reminder system monitors the mobile screen in consistency. The moment user gets within the radius of 500 meters, the reminder rings. The default radius range will be set to 500 meters that can be changed according to the users' desire. Not only the alarm, but its description and route map to the destination location is also provided.

\subsection{Set Timer Alarm}

In this module the timer is used to set an alarm that if the user wish to set a location for a particular time, then in this case the timer alarm is also set for that location.

\subsection{Remote Alarm}

In this module the user can set an alarm remotely on someone else mobile by whom the user is authorized for an access to this reminder system. This allows the user to remember his clerk, co-partners or friends for some getting some particular things

\section{CONCLUSION}

In this study, we have discussed and explored how we can optimally use the location-based services in our daily life. Location-based reminder system provides an efficient and user-friendly service to its user. In this location based alarm system based on the location of the mobile devices, the user will automatically get the notification in relevant situations allowing them easily to activate or deactivate the alarm system. Till now there were so many applications for reminding the work schedule which is working based on the alarm. But in our application, we have introduced a new thought for reminding the daily work remotely to another user if we authorized by that user.

\section{REFERENCES}

[1] G.V.M.vasuki, D.mounika ,CH.Dayeswari “ Location Based Alarm System Depending on Longitude and Latitude" (IJCSIT) International Journal of Computer Science and Information Technologies, Vol. 5 (1) , 2014.

[2] Teduh Dirgahayu, Feri Wijayanto Yogyakarta, Indonesia, "Location-based request forwarding in a geo-fencing application with multiple providers" , International Conference on Technology, Informatics, Management, Engineering \& Environment (TIME-E) 2015.

[3] Nur Rokhman; Lubab Saifuddin, "Location and time based reminder system on Android mobile device", 2nd International Conference on Science in Information Technology (ICSITech), 2016. 